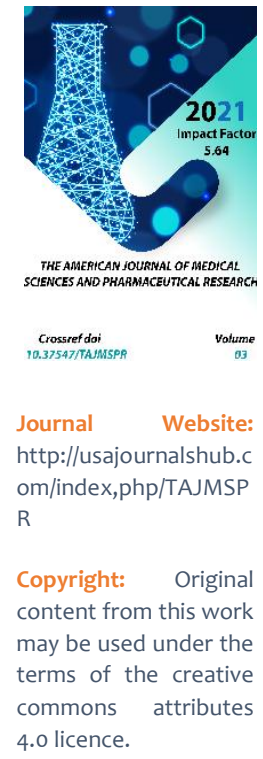

\title{
Early Prenatal Diagnosis And Prevention Of Birth Damage Development In Children (Spina Bifida - A Defect In The Development Of The Nervous System)
}

Makhbuba Mamatkulova

Senior Lecturer Of The Department Of Epidemiology And Infectious Diseases Of Fergana Medical Institute Of Public Health, Uzbekistan

\section{ABSTRACT}

Spina bifida-is a defect in the development of the neural tube, which is a splitting of the spinal canal and is one of the most common conqenital malformation of the fetus. Worldwidt, the incidence of fetal neural tube defects ranqes from 0,17 to 6,39 per 1000 newborns. This article describes methods for early diaqnosis of fetal neural tube malformation in earle preqnancy and the results of studies of preqnant woman at different staqes of preqnancy.

\section{KEYWORDS}

Spina bifida,neural defect,ultrasound screeninq, biochemical screeninq, PAPP-test,alphafetoprotein, chorionic qonadotropin $+\beta$, estriol, folic acid.

\section{INTRODUCTION}

On December 25, 2017, the President of Uzbekistan adopted the State program "Early detection of congenital and hereditary diseases in children." This program is designed for 2018-2022 to prevent the birth of children with these diseases. The most common congenital malformations - "Spina bifida" - a defect in the development of the neural tube, 
which is a splitting of the spinal canal (spinal dysraphism), often with the formation of hernias of the spinal cord. The relevance of prenatal diagnosis of spina bifida is associated with the severity of the pathology and the complexity of its correction. Spina bifida is one of the most difficult to diagnose congenital malformations of the neural tube with a multifactorial type of inheritance. The frequency of this defect depends on many factors, including geographic, ethnic and seasonal.

\section{GOALS AND OBJECTIVES OF THE WORK}

The goal is to prevent the birth of children with various developmental anomalies and genetically determined diseases. The upbringing of physically and mentally healthy children is considered the task of the family in front of society. However, in some cases, the fetus in the womb may not develop properly, and the child, whom the parents are expecting with great joy and excitement, is born with a disability or with hereditary diseases. To prevent such cases, it is safe and convenient to use the screening service. We, as doctors, have been convinced of this more than once.

Within the framework of the State program "Early detection of congenital and hereditary diseases in children", prenatal screening rooms were opened in the central multidisciplinary city and district polyclinics of the Fergana region and equipped with modern highresolution ultrasound scanners. The Republican Center for Mother and Child Screening of the Ministry of Health of the Republic of Uzbekistan is equipped with a hightech analyzer - a tandem mass spectrometer for diagnosing a wide range of hereditary diseases in newborns and young children.

MATERIALS AND METHODS
As a result of the measures taken, 1.1 million pregnant women were examined, which made it possible to prevent the birth of over 21 thousand children with congenital malformations. More than 1.7 million newborns were examined for hereditary and congenital diseases, 2.7 thousand children received qualified medical assistance to eliminate congenital malformations.

\section{RESEARCH METHODS}

Ultrasound screening provides for at least 3fold examination of the fetus:

-10-14 weeks

- 20-24 weeks

- 30-32 weeks

When examined at 10-14 weeks, gross developmental defects can be detected anencephaly, omphalocele, acrania, exencephaly, cervical hygroma and some others.

At 20-24 weeks, ultrasound can detect most of the gross anatomical anomalies of development - defects of the brain and spinal cord, kidneys, facial clefts, gross heart defects, developmental defects of the limbs, gross abnormalities of the gastrointestinal tract. Most of the developmental abnormalities detected in mid-pregnancy cannot be treated with surgery and are a medical indication for termination of pregnancy. Also at this stage, the presence of markers of fetal chromosomal pathology is assessed, which include: high and low water, fetal growth retardation, enlargement of the renal pelvis (pyeloectasia), expansion of the ventricles of the brain (ventriculomegaly), a decrease in the size of the nasal bone of the fetus, shortening of the length of tubular bones, hyperechoic intestines, hyperechoic inclusions in the heart of the fetus, cysts of the choroid plexus of the brain and a number of others. 
At the stage of 30-32 weeks, developmental anomalies with low anatomical severity and late manifestation can be detected - heart defects, hydrocephalus, obstruction (narrowing) of the urinary tract. Many of these developmental anomalies are subject to surgical correction after the baby is born.

Correctly organized mass ultrasound screening allows to reveal the majority of gross anatomical defects before the fetus reaches the age of viability. But ultrasound has its limits of information content. Ultrasound cannot detect a number of common genetic disorders of the fetus that do not have significant anatomical manifestations, in particular, chromosomal diseases (including Down's syndrome).

For a complete examination of pregnant women, a combined screening is carried out - a combination of ultrasound and biochemical screenings.

Biochemical screening by analyzing the blood of pregnant women for serum markers serves as a means of forming a risk group for the chromosomal pathology of the fetus. The fetoplacental complex, consisting of the fetus and fetal membranes (chorion transforming into the placenta, the water membrane amnion) produces specific proteins that penetrate into the blood of a pregnant woman. Changes in the state of the fetoplacental complex, occurring for various, including genetic reasons, are reflected in the level of specific proteins (serum markers).

Currently, biochemical screening is carried out in two stages - screening for the first trimester (10-13 weeks) and screening for the second trimester (16-20 weeks).In the first trimester (10-13 weeks), a PAPP-A test is performed. PAPP-A is a high molecular weight glycoprotein. A number of serious clinical studies indicate the diagnostic value of PAPP-A as a screening marker for the risk of fetal chromosomal abnormalities. PAPP-A levels are significantly reduced when the fetus has trisomy 21 (Down's syndrome) or trisomy 18 (Edwards syndrome). In addition, this test is also informative in assessing the threat of miscarriage and stopping pregnancy at a short time.

In the second trimester of pregnancy, an AFP / hCG test is performed. Alpha-fetoprotein (AFP) is one of the indicators of the general condition of the fetus and the likelihood of having a congenital pathology. Elevated concentrations of AFP in maternal serum or amniotic fluid during pregnancy may indicate congenital spinal degeneration, anencephaly, esophageal closure, or multiple pregnancies.

In the Fergana Regional Screening Center "Mother and Child", the blood serum of pregnant women is examined simultaneously for AFP, CG and estriol.

Chorionic gonadotropin $+\beta($ hCG $+\beta)$ - is produced in the placenta during pregnancy. Elevated levels indicate choriocarcinoma, gallbladder drift, or multiple pregnancies. A reduced value indicates a threat of miscarriage or miscarriage, ectopic pregnancy, preeclampsia, or intrauterine death.

Estriol is the predominant estrogen in the blood and urine of pregnant women. The combined determination of unconjugated estriol, chorionic gonadotropin (hCG) and alpha-fetoprotein (AFP) in the second trimester of pregnancy is a study useful, along with other clinical data of the mother, in assessing the risk of fetal chromosomal abnormalities at birth.

Screening is currently recommended for the following types of congenital malformations in the fetus: 
- $\quad$ Down syndrome (trisomy on 21 pairs of chromosomes)

- $\quad$ Edwards syndrome (trisomy on 18 pairs of chromosomes)

- Neural tube defects (hernia of the spinal cord, anencephaly).

The most common congenital malformations "Spina bifida" - a defect in the development of the neural tube, which is a splitting of the spinal canal (spinal dysraphism), often with the formation of hernias of the spinal cord.

The central nervous system is formed from the ectoderm at the 3rd week of embryonic development. The embryo length during this period is only $15 \mathrm{~mm}$. The ectoderm leaves come together and form a neural groove, and then, approaching, a neural tube. A bony ring is formed around. Normally, the closure of the neural tube occurs by the 28th day of gestation. However, with violations of the closure, such defects as anencephaly, encephalocele and spina bifida are formed.

Typical for all types and forms of developmental defect of the neural tube is their posterior location with a defect in the posterior semicircle of the spinal canal. Very rarely, less than $1 \%$ of cases, a non-closure is formed on the anterolateral surface of the canal, and anterior spinal hernias occur.

The anterior and posterior clefts of the vertebra can run along the midline, and also be located asymmetrically. In some cases, the gap is oblique. If the splitting of the vertebrae occurs along the midline, then the deformity of the spine may be insignificant or not at all pronounced. However, with an oblique and asymmetric location of the gap in combination with other anomalies in the development of the vertebrae (for example, one-sided microspondilia of a half of the vertebra, an anomaly of the articular processes), severe deformity of the spine develops. Most often (up to $70 \%$ of cases) spina bifida is localized in the lumbosacral region, in $21 \%$ - in the thoracic region and in $9 \%$ - in other localization.

On July 17, 2018, the Ministry of Health of the Republic of Uzbekistan adopted the "Regulations on the procedure for preventing the birth of children with birth defects that impede the health and survival of the fetus." According to the appendix to this charter, spina bifida in the cervical, thoracic and lumbosacral regions with hydrocephalus is determined by ultrasound screening in the second trimester of pregnancy and is considered an uncorrected defect. Spina bifida in the cervical, thoracic and lumbosacral regions without hydrocephalus is detected on ultrasound screening in the second trimester of pregnancy and is considered a correctable defect.

There are three types of spina bifida:

Spina bifida occulta - "hidden" spina bifida. In this version, there is no visible external defect. Latent spinal clefts are usually localized in the lumbosacral region and, as a rule, do not manifest themselves clinically. Often they are an accidental "find" in the X-ray examination of the spine or MRI. The anatomical essence of hidden spina bifida consists in incomplete fusion of the vertebral arch. This is the most favorable variant of the spina bifida. Sometimes in the area of the defect there are hyperpigmentation and hair tufts. In such cases, the child has spinal cord abnormalities below the affected area: lipomas and abnormal fixation of the spinal cord.

With closed defects, vertebral anomalies, foot deformities and an abnormally low-lying cone are also found. The closed variant of spina bifida is not accompanied by Arnold Chiari II syndrome, ventriculomegaly and other intracranial changes 
Meningocele - meningocele is a spina bifida with protrusion into a defect in the dura mater, but without the involvement of nerve structures in the process. The contents of the hernial sac are the meninges and the cerebrospinal fluid, its shape is usually stalked with a narrowed leg. The bone defect usually covers 2-3 vertebrae. Clinical manifestations of the disease are variable and range from asymptomatic course to dysfunction of the pelvic organs, movement and sensory disorders. This variant of spina bifida is rare.

\section{RESULTS AND DISCUSSION}

Meningomyelocele - meningomyelocele is the most severe form of spina bifida, involving the membranes, spinal cord and its roots in the hernial sac. The bone defect is usually wide and
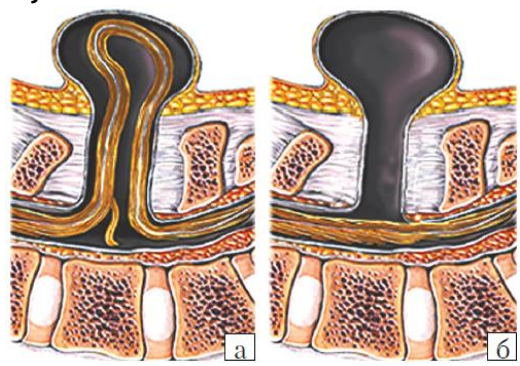

Fig. 1. Scheme of fetal spine defects.

Scheme of fetal spine defects: a -

Clinical observation 1 meningomyelocele, $\mathrm{b}$ - meningocele

a) Meningomyelocele.

b) Meningocele.

We present clinical observations demonstrating the capabilities of ultrasound in the diagnosis of fetal spine defects. extended, covering from 3 to 6-8 vertebrae. The degree of the neurological defect is always severe paraplegia of the lower extremities, sensory disorders, neurogenic bladder and intestinal paresis. It is this form of spinal hernias that occurs most often - about $75 \%$ of all forms. In almost all cases, meningomyelocele is combined with ArnoldChiari II syndrome. Thus, the detection of signs of an Arnold-Chiari II anomaly in the fetus is a marker of the presence of spina bifida. In addition, in $70-80 \%$ of cases the fetus develops hydrocephalus (Fig. 1). 
violations of the structures and integrity of tissues were found (Fig. 2).

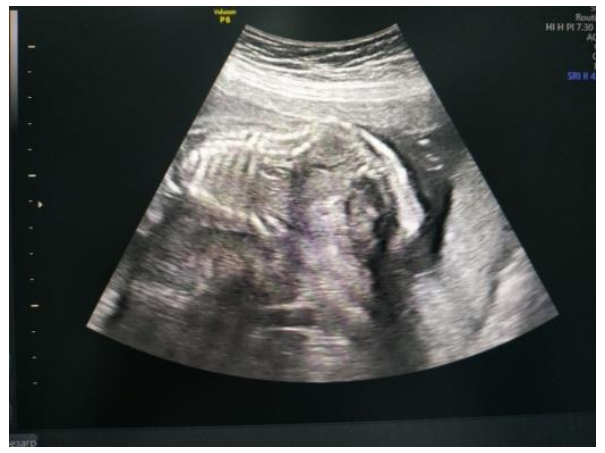

Fig. 2.

\section{Clinical observation 2}

Patient M., 35 years old, applied to the prenatal office at 14-15 weeks of pregnancy. The study was carried out on a GE Voluson P6 apparatus using the 3D / 4D surface volume reconstruction mode. During scanning of the spine in the frontal and sagittal planes, angular deformity of the spine in the lumbar spine was revealed. In the transverse scanning plane, violations of the structures and integrity of tissues were found (Fig. 3).

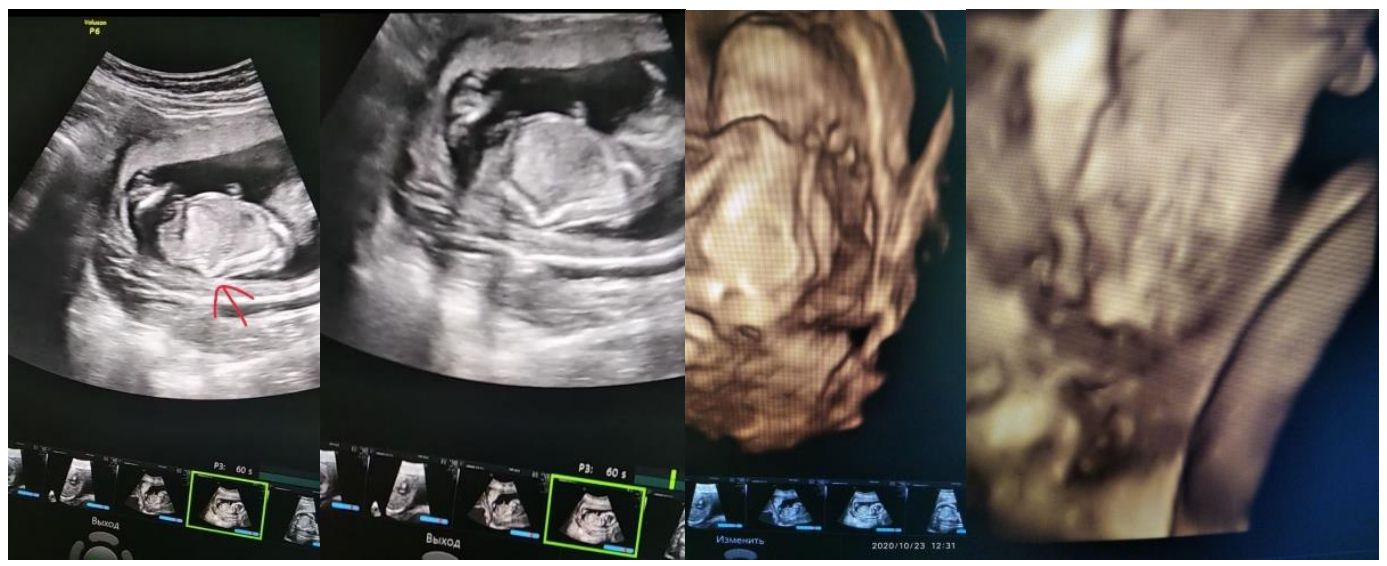

Fig. 3

Clinical observation 3

Patient N., 36 years old, came to the prenatal office at 14 weeks of pregnancy. The study was carried out on a GE Voluson P6 apparatus using the 3D / 4D surface volume reconstruction mode. In the process of scanning the spine in the frontal and sagittal planes, angular deformity of the spine in the lumbar spine was revealed (Fig. 4, 5). 


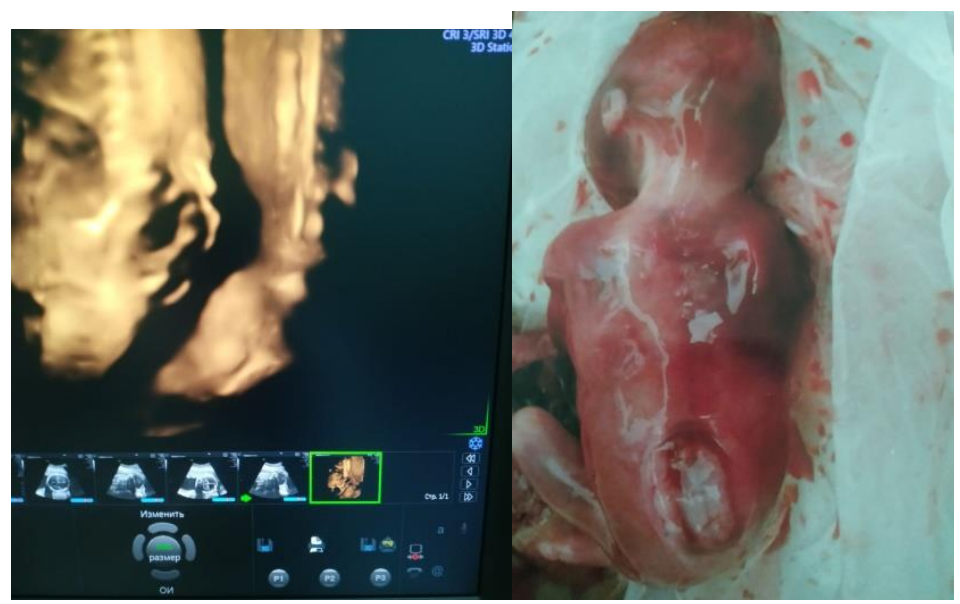

Fig. 4 .

Fig. 5. Abortion phenotype at 15 weeks.

Clinical observation 4

Patient F., 26 years old, came to the prenatal office at 22 weeks of pregnancy. The study was carried out on a GE Voluson P6 apparatus using the 3D / 4D surface volume reconstruction mode. Fetometry indices fully corresponded to the gestational age. During scanning of the spine in the frontal and sagittal planes, angular deformity of the spine in the lumbar spine was revealed. (fig. 6).

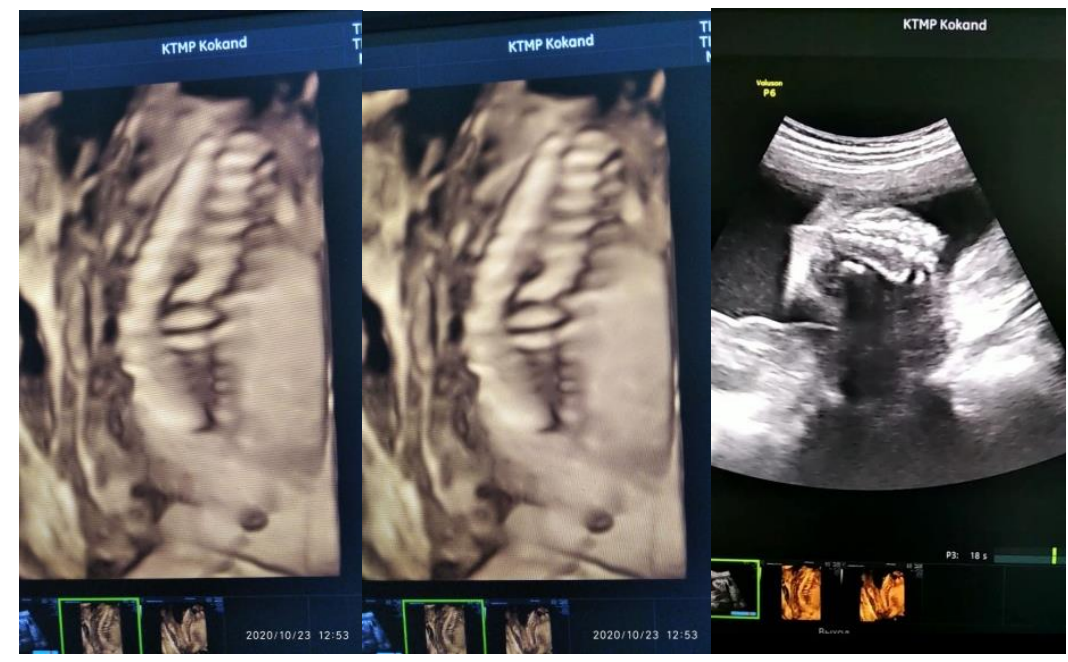

Fig. 6.

Clinical observation 5

Patient I., 22 years old, applied to the prenatal office at 17 weeks of pregnancy. The study was carried out on a GE Voluson P6 apparatus using the 3D / 4D surface volume 
reconstruction mode. Fetometry indices fully corresponded to the gestational age. In the process of scanning the spine, an angular deformity of the spine in the lumbar spine was revealed. In the transverse scanning plane, violations of the structures and integrity of tissues were found (Fig. 7).

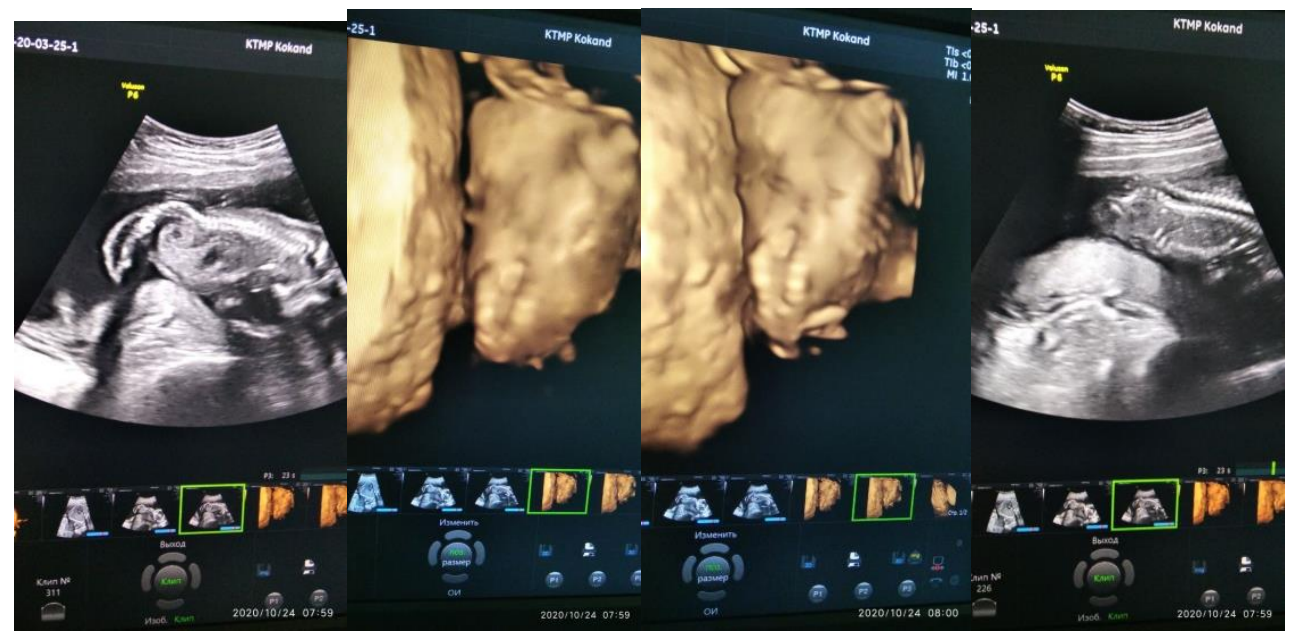

Fig. 7.

Differential diagnosis and justification for additional research.

\begin{tabular}{|l|l|l|l|}
\hline Diagnosis & $\begin{array}{l}\text { Rationale for differential } \\
\text { diagnosis }\end{array}$ & Surveys & $\begin{array}{l}\text { Diagnosis exclusion } \\
\text { criteria }\end{array}$ \\
\hline Spinal hernia & $\begin{array}{l}\text { violation of the } \\
\text { formation of the spinal } \\
\text { canal with the formation } \\
\text { of bony septa and spinal } \\
\text { cord splitting }\end{array}$ & $\begin{array}{l}\text { On radiography a } \\
\text { violation of the } \\
\text { formation of the spinal } \\
\text { canal with the formation } \\
\text { of bony septa and spinal } \\
\text { cord splitting }\end{array}$ & $\begin{array}{l}\text { They reach large sizes, } \\
\text { soft consistency, } \\
\text { pulsation, fluctuation } \\
\text { can be determined. The } \\
\text { color of the hernia is } \\
\text { bluish. }\end{array}$ \\
\hline Teratoma & no splitting & $\begin{array}{l}\text { There is no splitting on } \\
\text { X-ray of the spine. }\end{array}$ & $\begin{array}{l}\text { Lobularity of the } \\
\text { structure, the presence } \\
\text { of dense inclusions and } \\
\text { an asymmetric location } \\
\text { of the tumor. }\end{array}$ \\
\hline
\end{tabular}

Treatments: The main goal of surgical treatment in patients with spinal hernia is to remove the hernial sac and plastic defect in the arches of the vertebrae, to reduce or stabilize neurological deficit, to restore physiological barriers.

Microsurgical excision of a spinal hernia. 
Deformity and diseases of the spine (congenital and acquired), ineffectiveness of conservative treatment, severe pain syndrome, cosmetic defect is an indication for surgery.

Contraindications to surgery are infection and development of meningitis, meningoencephalitis, purulent - inflammatory processes in the nervous system, severe malformations of the spinal cord and spine; Surgical intervention is accompanied by difficulties in carrying out plastics of the spinal canal due to the perineum and the width of the defect.

Further management in the postoperative period: activating the patient on days 2-3 after the operation, learning to walk and adapting after the operation, control CT or MRI of the spine on an outpatient basis at 6 and 12 months after the operation, undergoing rehabilitation up to 6 months after the operation, follow-up period: 2 once a year at a neurosurgeon doctor at the place of residence and exercise therapy, massage.

The first stage (early) of medical rehabilitation is the provision of MR in inpatient conditions (resuscitation and intensive care unit or specialized specialized department) from the first 12-48 hours in the absence of contraindications. The patient's stay at the first stage ends with an assessment of the severity of the patient's condition and disorders in accordance with international criteria and the appointment by the coordinator of the next stage of the MR.

Indicators of the effectiveness of treatment and the safety of diagnostic and treatment methods described in the protocol:

Lack of impaired mobility of the lower extremities;
Restoration of sensitivity, function of the pelvic organs;

\section{Maintaining a normal neurological status in the preoperative period.}

Improving the general condition of the patient

Regression of neurological symptoms;

Postoperative wound healing.

\section{CONCLUSION}

For 9 months of 2019, 23 children with this diagnosis were born in the Fergana region. Comparative statistics show that for 9 months of 2020, the number of births with this diagnosis decreased, and 5 children were born with this diagnosis. The frequency of spin bifida is, according to various sources, 1-2 cases per 1,000 newborns. The frequency of repeated births with this defect is $6-8 \%$, which confirms the role of genetic factors in the onset of the disease. Most often, the diagnosis under consideration occurs in children from mothers of considerable age. 95\% of newborns with spina bifida are born to parents without such a diagnosis.

Currently, the greatest interest is the identification of this defect in the first trimester of pregnancy. Ultrasound criteria such as lack of intracranial translucency, smoothness of the brainstem angle, decrease in biparietal size below the 5th percentile, etc. are being studied. Thus, the diagnosis of spina bifida remains an important task in prenatal diagnosis.

\section{REFERENCES}

1. Resolution of the President of the Republic of Uzbekistan "On the State Program for the Early Detection of 
Congenital and Hereditary Diseases in Children for the Period 2018-2022"

2. Ministry of Health of the RUz No. 40-A "Regulations on the procedure for preventing the birth of children with birth defects that impede the health and survival of the fetus."

3. Inamova S.T., Karimov A.Kh., Fazylova S.A., Rasulova M.M., Nasritdinov H.K., Kamalidinova Sh.M. Ultrasound technology in obstetrics. T .: Fan, 2012.

4. Romero R., Peelu J., Genti F. et al. Prenatal diagnosis of congenital malformations of the fetus. Per. from English $M$.: Medicine, 1994.

5. Medvedev M.V. Prenatal diagnosis of congenital malformations in early pregnancy. 2005.

6. Medvedev M.V. Fundamentals of ultrasound screening at 18-21 weeks of gestation: a practical guide for doctors. $M$ $\therefore$ Real Time, 2013.

7. Abukhamad, R.Shaui. Ultrasound diagnosis of fetal anomalies in the first trimester of pregnancy. Per. From eng. E.V. Yudina. R.: Vidar-M, 2019. 